\title{
Meanline Analysis of Turbines with Choked Flow in the Object-Oriented Turbomachinery Analysis Code
}

\author{
Eric S. Hendricks* \\ NASA Glenn Research Center, Cleveland, Ohio, 44135
}

\begin{abstract}
The prediction of turbomachinery performance characteristics is an important part of the conceptual aircraft engine design process. During this phase, the designer must examine the effects of a large number of turbomachinery design parameters to determine their impact on overall engine performance and weight. The lack of detailed design information available in this phase necessitates the use of simpler meanline and streamline methods to determine the turbomachinery geometry characteristics and provide performance estimates prior to more detailed CFD analyses. While a number of analysis codes have been developed for this purpose, most are written in outdated software languages and may be difficult or impossible to apply to new, unconventional designs. The ObjectOriented Turbomachinery Analysis Code (OTAC) is currently being developed at NASA Glenn Research Center to provide a flexible meanline and streamline analysis capability in a modern object-oriented language. During the development and validation of OTAC, a limitation was identified in the code's ability to analyze and converge turbines as the flow approached choking. This paper describes a series of changes which can be made to typical OTAC turbine meanline models to enable the assessment of choked flow up to limit load conditions. Results produced with this revised model setup are provided in the form of turbine performance maps and are compared to published maps.
\end{abstract}

\section{Nomenclature}

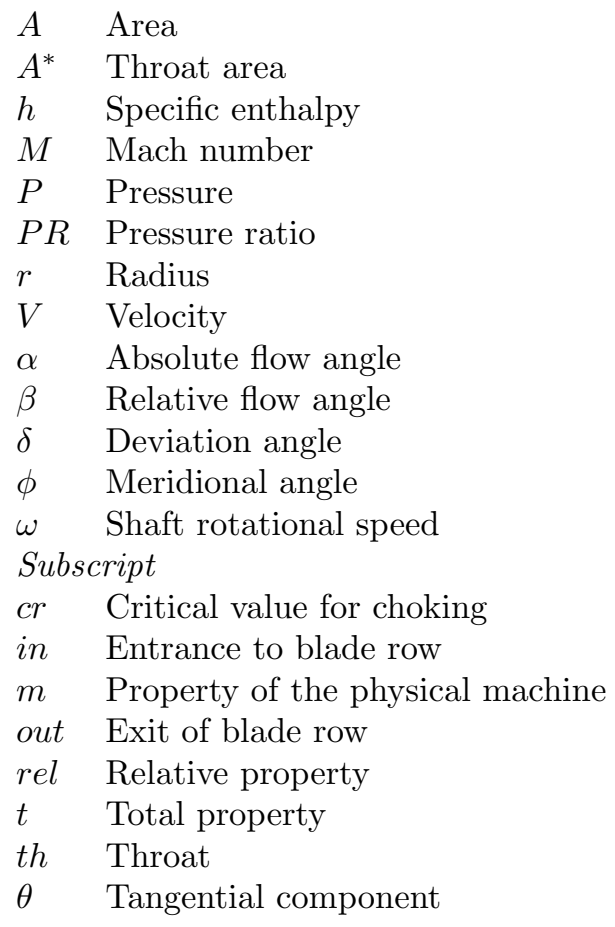

*Aerospace Engineer, Propulsion Systems Analysis Branch, MS 5-11. 


\section{Introduction}

PREDICTIOn of turbomachinery performance characteristics is an important part of the gas turbine engine $P$ design process. The engine design process begins with a conceptual design phase focused on determining the cycle performance and geometric characteristics assuming reasonable estimates for component performance levels. As the process advances to preliminary design, refined estimates for the overall performance and geometric parameters are generated by developing initial designs for each of the engine components. In this process, the designer faces the challenge of developing the component design using an increasingly large number of component design parameters while hoping to match or exceed the assumed performance levels from conceptual design.

To support the conceptual/preliminary design of each engine component, lower-fidelity analysis tools are often employed to determine the macro design parameters prior to advancing to more detailed, higher-fidelity analyses. For turbomachinery components, the lower-fidelity conceptual design process usually consists of meanline and streamline analyses. While a number of these analysis tools have been developed, most of them are challenging to use as they are written in outdated software languages and may have little supporting documentation. Furthermore, these analysis tools were often written with a limited design space in mind making them unsuitable for application to new turbomachinery architectures or unique configurations.

To address these challenges, a new meanline and streamline turbomachinery analysis code is being developed by NASA Glenn Research Center. The Object-Oriented Turbomachinery Analysis Code (OTAC) ${ }^{1}$ is developed using the interpreted language features available in the Numerical Propulsion System Simulation (NPSS). ${ }^{2}$ In addition to using a modern object-oriented software language, building OTAC within NPSS provides access to thermodynamic property packages and a sophisticated Newton-Raphson solver. Using these features, new objects have been created to represent blade rows, blade sections, transition ducts, expanders and reducers. These objects can then be combined to form a wide variety of meanline and streamline turbomachinery models including both axial and centrifugal compressors and turbines. OTAC's meanline and streamline modeling capabilities have been validated by comparing results from a number of compressor and turbine models to other results from other analysis tools. ${ }^{3}$

While the validation results for OTAC compare well to other analysis tools, a limitation of OTAC was identified when analyzing turbines. This limitation occurs when attempts are made to analyze the turbine with operating conditions near or at the limiting choked mass flow rate, ultimately resulting in the analysis failing to converge. ${ }^{3}$ Addressing this limitation is critically important for the analysis of turbines as cycle operating points (match points) typically fall within the choked region of the turbine performance map. Designing a turbine to operate in this choked flow region of the map is necessary in order to obtain higher values of specific power per stage.

Given the importance of analyzing turbines with choked flow, a method which can be applied to OTAC turbine meanline models to enable assessment of choked flow cases was developed and is presented in this paper. The next section describes the developed OTAC components in more detail along with the setup of a typical OTAC turbine mealine analysis. Following this description, Section III describes the relevant supersonic flow characteristics which must be captured by the meanline model. Section IV then presents improvements which can be implemented in OTAC meanline models to enable the analysis of both subsonic and supersonic flow. Section V presents the results of OTAC meanline analyses using the described improvements and compares the results to those published in literature. Finally, conclusions from the current work are presented along with areas for future improvement.

\section{Typical OTAC Turbine Meanline Model Setup}

The Object-Oriented Turbomachinery Analysis Code is a relatively new tool for meanline and streamline analysis of both compressor and turbine components. The object-oriented structure of the code provides flexibility allowing it to be applied to a wide variety of conventional and unconventional turbomachinery concepts. Additionally, the flexibility enables customization by each user to apply the appropriate or preferred loss calculations for the system under assessment. This section will summarize the relevant OTAC objects needed to construct a turbine meanline model and describe how a typical model is setup. The reader is referred to the publications by Jones ${ }^{1,3}$ for additional details. 


\section{Overview of OTAC Objects}

The development of OTAC created 6 new objects using the NPSS interpreted language capability: OTACstart, TransitionSegment, BladeRow, BladeSegment, Expander, and Reducer. In addition to these objects, the Shaft and FlowEnd objects from NPSS are used in the construction of OTAC models. Of the new OTAC objects, the Expander and Reducer objects are only required for a streamline analysis and will therefore not be discussed in this paper. The OTACstart object is used to initiate the gas flow at the entrance of the turbomachinery model, typically by specifying the mass flow rate, total pressure, total temperature, and absolute flow angle. The TransitionSegment is a relatively simple object which allows for changes to the annulus geometry (mean radius and area) between blade rows of a turbomachine. The remaining two objects, the BladeRow and BladeSegment, are at the core of the OTAC analysis and are discussed in more detail below.

The BladeRow and BladeSegment objects work together to model the flow as it passes through a turbomachinery blade row. BladeSegment focuses on computing the changes to fluid properties along a single streamline as it passes through the blade. It is important to note that the BladeSegment object does not represent a fixed spanwise section of the blade, but actually represents a streamtube (with a streamline at its midspan) as it passes through the blade. Therefore the radial location of the BladeSegment may vary with changes to the flow properties. The BladeRow object represents an entire blade row and contains one or more blade segments. In a streamline analysis, simple radial equilibrium is enforced to determine the location of each BladeSegment.

The calculations in the BladeSegment assume the entrance flow conditions are known allowing for the exit flow properties to be determined. The exit flow properties are determined by ensuring that the seven equations below are satisfied:

$$
\begin{gathered}
\dot{m}_{\text {in }}=\dot{m}_{\text {out }} \\
h_{t, \text { out }}-h_{t, \text { in }}=\omega\left(r_{\text {out }} V_{\theta, \text { out }}-r_{\text {in }} V_{\theta, \text { in }}\right) \\
P_{t, \text { out }}=P_{t, \text { out }, \text { ideal }}-f_{\text {loss }}(\text { args }) \\
\beta_{\text {out }}=\beta_{m, \text { out }}+\delta \\
r_{\text {out }}=r_{m, \text { out }} \\
A_{\text {out }}=A_{m, \text { out }} \\
\phi_{\text {out }}=\phi_{m, \text { out }}
\end{gathered}
$$

The first equation ensures mass is conserved as the streamtube passes through the blade. Equation 2 is the Euler turbomachinery equation for flow along a streamline. Equation 3 sets the exit total pressure by applying losses to the ideal exit pressure. The loss calculations are complex and are represented in this equation by a generic function; the object-oriented structure of OTAC allows for any loss model to be inserted into this function as long as it matches one of the available loss definitions. Equation 4 ensures that the exit flow angle matches the blade metal angle with an adjustment for deviation. The final three equations guarantee that the flow mean radius, area and meridional angle must match those of the physical machine.

In order to satisfy these equations (commonly referred to in NPSS as dependent conditions), a NewtonRaphson non-linear solver is applied within each BladeRow object. Equations 1 and 7 are typically trivially solved and therefore excluded from the non-linear solution process. The remaining five dependent conditions for meanline analysis require selecting five independent parameters which can be varied to converge the equations. The independent parameters for this solution process are $M_{\text {out }}, \alpha_{\text {out }}, P R, h_{t, \text { out }}$, and $r_{\text {out }}$. By varying these independent parameters, the dependent equations can be satisfied thereby determining the flow properties at the exit of the blade row. 


\section{Constructing OTAC Meanline Models}

OTAC models compressors and turbines by combining the previously described objects to match the desired turbomachinery architecture. For the meanline analysis of a single-stage turbine, a typical OTAC model structure would be constructed by instantiating and connecting the objects as shown in Fig. 1. First, an OTACstart would be used to initiate the flow through the model. The flow would then be passed to a BladeRow representing a stator (or vane) with a single BladeSegment. The flow may then pass through a TransitionSection before entering another BladeRow representing a rotor. This rotor is connected mechanically to a NPSS shaft object. The flow from the rotor may then connect to another transition section before terminating in a FlowEnd. The model setup shown in Fig. 1 is representative of most models of a single-stage turbine. Different turbomachinery architectures and concepts can be easily produced by adding or removing instances of each object.

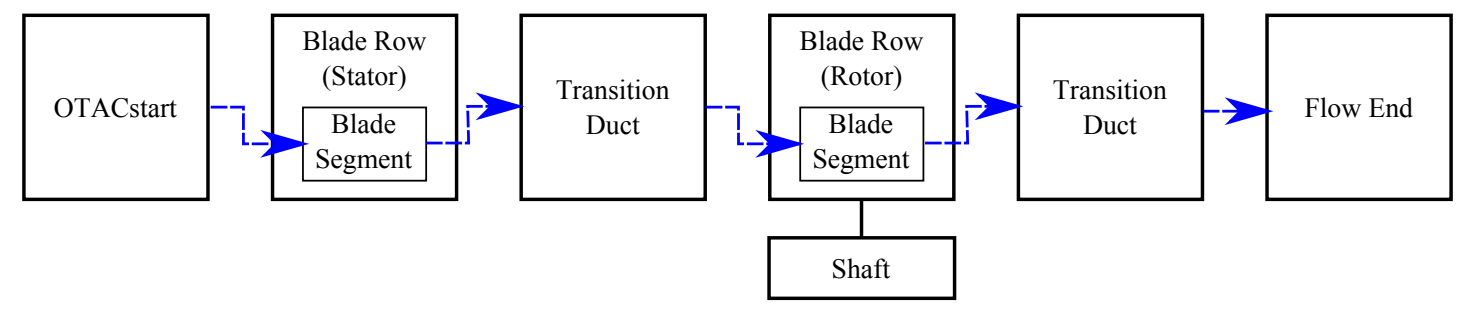

Figure 1. Object Layout of a Typical Single-Stage Turbine Meanline Model.

Once the model has been created by instantiating and connecting the various objects, OTAC is first executed in a design mode to determine the turbine characteristics based on a set of design inputs. Once the turbine design is established in this mode, additional operating conditions can be analyzed in an off-design mode by specifying inlet flow properties such as total pressure, total temperature, mass flow rate, and flow angle as well as the shaft speed. During the design and off-design model execution, a Newton-Raphson non-linear solver is created within each BladeRow object as previously mentioned with independents and dependents to solve Equations 2 through 6. Furthermore, a top-level Newton-Raphson solver is automatically created within the model to allow for additional dependent conditions to be converged that do not fit within an individual BladeRow solver. In the default OTAC setup however, this top-level solver is empty. A summary of the case inputs and solver setup for a typical OTAC meanline model in off-design mode is given in Fig. 2.

\section{Relevant Turbine Flow Characteristics}

In order to improve the off-design analysis within OTAC turbine models, it is important to first have a clear understanding of the relevant turbine flow phenomena. This section first describes the the flow characteristics that occur within a blade row when that blade row chokes. Second, the effect of flow angle on the isentropic Mach-area relationship is examined.

There are several important changes that occur in the flow characteristics when supersonic conditions are encountered in a turbine blade row. Figure 3 depicts a meanline cut through several turbine blade rows along with important features of the flow field. The green line in the figure represents the throat or minimum area of the blade passage. The throat may occur anywhere within the blade passage but is commonly found near or at the trailing edge of the blade. The total throat area, determined by multiplying the individual passage throat area by the number of blades, limits the air flow that can pass through that particular blade row. Similar to a nozzle, the mass flow through the turbine blade row chokes when the ratio of inlet relative total pressure $\left(P_{t, r e l, i n}\right)$ to exit static pressure $\left(P_{s, o u t}\right)$ exceeds the critical value for sonic flow to occur at the throat. When this total-to-static pressure ratio exceeds the critical value, additional expansion of the flow is required downstream of the throat in order to match the exit static pressure. This expansion will occur through a complex combination of Prandtl-Meyer expansion and shock waves. The location of the expansion and shock waves is highly dependent on the detailed blade geometry and beyond the scope of meanline analysis. However, the effect of passing through the expansion and shock waves is that the flow exiting the 


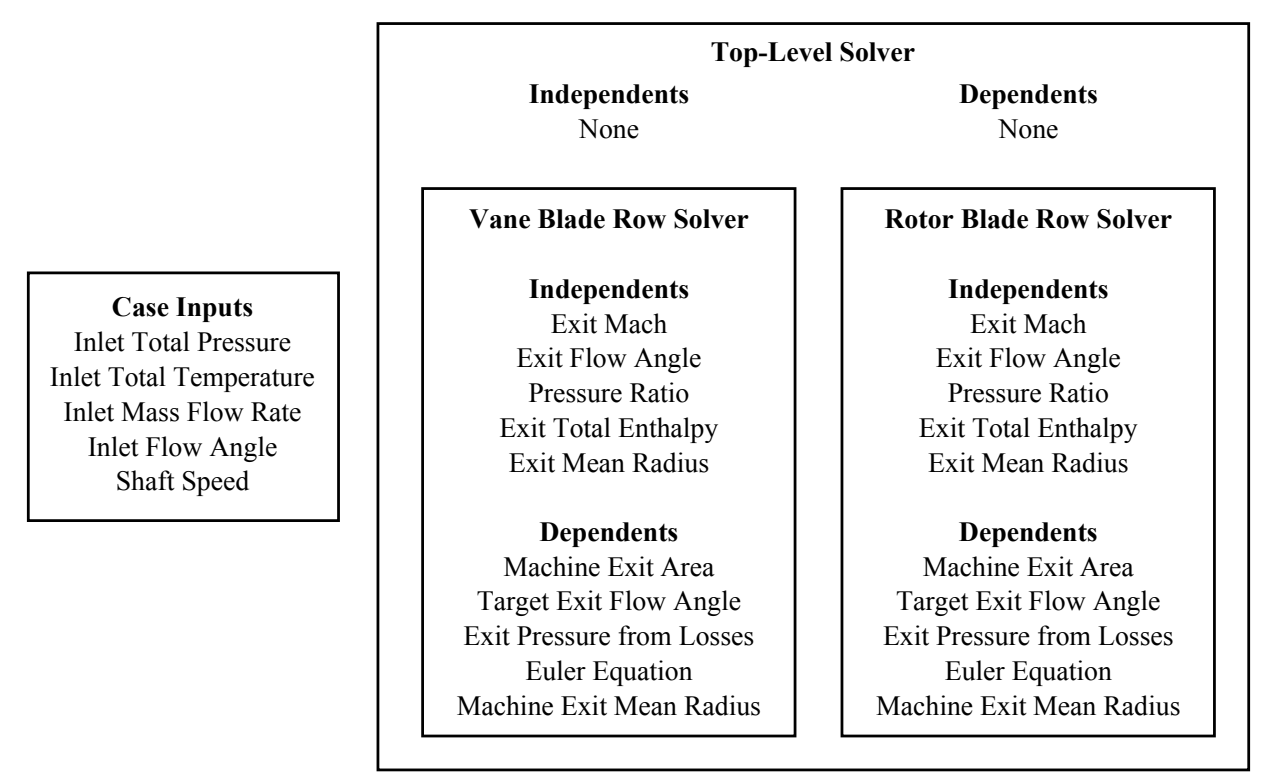

Figure 2. Case Inputs and Solver Setup for a Typical Single-Stage Turbine Model.

blade row will deviate from the blade metal angle to match the blade exit static pressure. ${ }^{4}$ Expansion to match the blade row exit static pressure is further constrained by choked flow in the downstream annulus. This choking occurs when the axial component of the exit flow Mach number becomes sonic. This case serves as a good approximation of the turbine limit load which is defined the maximum power that can be extracted by the turbine. ${ }^{5}$

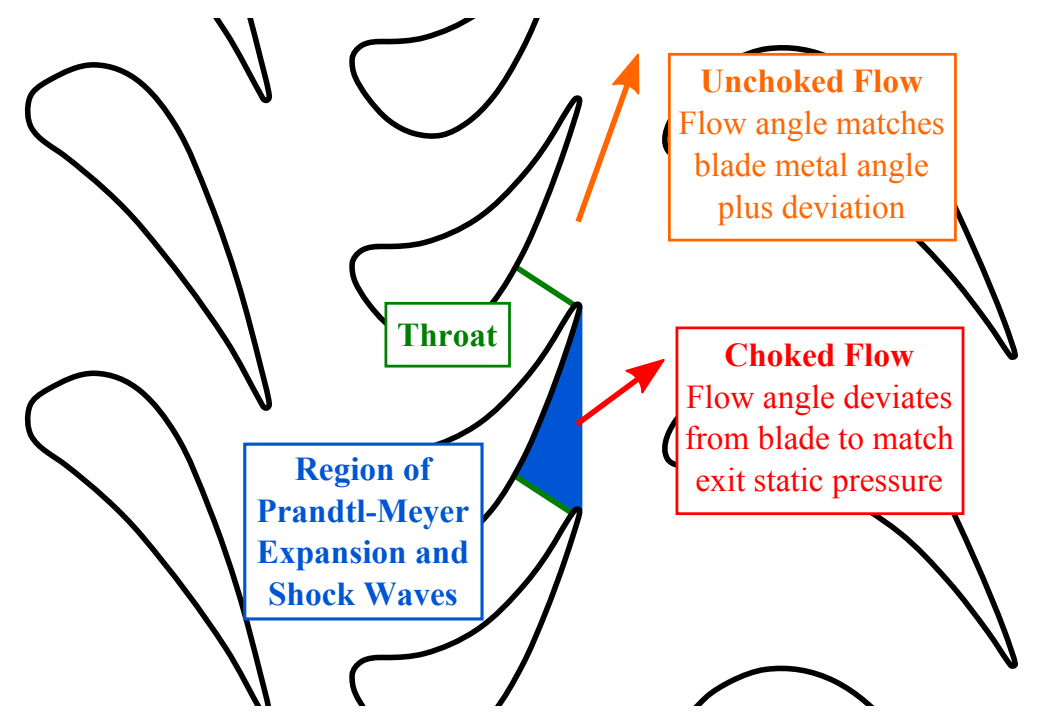

Figure 3. Effect of Choking on Exit Flow Angle.

These changes to the exit flow from the blade row can also be viewed in terms of the Mach-area relationship. For isentropic flow in a 1D duct with an assumed mass flow rate, total pressure, total temperature and ratio of specific heats there is a unique relationship between the duct area and the flow Mach number. If this duct is instead an annulus with the flow having an angle relative to the axial direction of $\alpha$, the relationship between area and Mach number is also effected by the flow angle as shown in Fig. 4 . Here, $A^{*}$ is the minimum area required to pass the specified flow with zero swirl angle. This figure is important as it 
shows how the flow angle must be reduced in order to match a specified flow rate as the exit relative Mach number increases from 1.

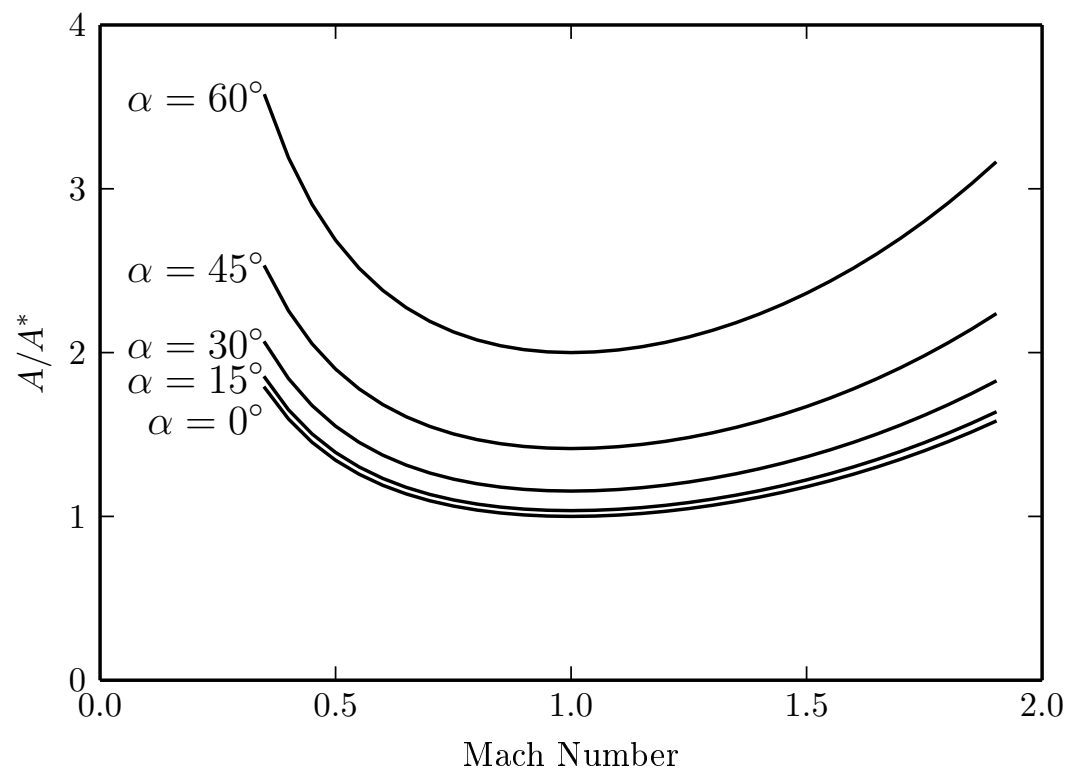

Figure 4. Mach-Area Relationship as Function of Flow Angle ( $\alpha$ ).

\section{Improvements to OTAC for Choked Flow}

While the default OTAC objects and typical model setup described in the first section work well for turbines with subsonic flow, significant limitations were encountered when trying to apply the analysis tool to turbine with supersonic relative Mach numbers. Of primary concern, the default BladeRow object does not properly capture the supersonic flow characteristics as the component lacks calculations to determine the allowable mass flow through the blade passage throat. Additionally, the equations solved by these objects constrain the flow to match a prescribed exit angle set by the blade metal angle and a deviation model. While a deviation model could theoretically be created which captures the proper behavior, it would be exceedingly difficult to generate a model which provided the exact exit flow angle for all potential analysis cases. Lastly, the model requires the user to input the turbine mass flow for each off-design case. This setup is not ideal as the user may provide a mass flow rate beyond the choked limit resulting in an unconverged case. Even if a flow rate exactly matching that of choking were specified, the model would still have difficulty converging as there are potentially an infinite number of solutions for that combination of case inputs. In order to address these limitations, three significant changes described in the sections below were made to OTAC.

\section{Changes to the Blade Row for Throat Calculations}

The first change made to OTAC was to add calculations to each BladeRow object in the model to calculate the throat area (in design mode) and an allowable mass flow rate constraint. Both of these calculations start by determining the critical and actual total-to-static pressure ratios across the blade row using Equations 8 and 9 respectively.

$$
\begin{gathered}
P R_{c r}=P_{t, r e l, \text { in }} / P_{s, t h, M=1.0} \\
P R=P_{t, \text { rel }, \text { in }} / P_{s, \text { out }}
\end{gathered}
$$


If the actual pressure ratio exceeds the critical pressure ratio, the flow through the blade row is choked. If the blade row is choked, the flow properties and area at the throat are computed by setting a sonic throat Mach number. When the pressure ratio is less than the critical pressure ratio, the throat flow properties and area are determined based on the exit static pressure. If the OTAC model is being executed in design mode, the calculated throat area becomes the design area and is held constant when evaluating the turbine at off-design conditions.

Next, calculations are added for the off-design mode to determine an allowable mass flow rate constraint. The mass flow rate constraint is formed by calculating the actual mass flow rate per unit throat area and comparing that value to the flow per unit area which would be required to choke the specified mass flow rate. The actual value is calculated by simply dividing the mass flow rate by the throat area computed in design mode. The required flow per area for choking is computed by dividing the mass flow rate by the area required to choke the flow. This required value must be less than or equal to the actual value forming the constraint defined in Equation 10. This constraint equation for the allowable flow rate can be applied in OTAC through a feature of the NPSS Newton-Raphson solver and will be discussed as part of the Solver Setup section.

$$
\frac{W}{A_{c r}} \leq \frac{W}{A_{t h}}
$$

\section{Changes to the Case Inputs}

In addition to changes to the OTAC objects themselves, changes were also necessary in the case inputs. The objective of these changes was to remove the mass flow rate as an input and allow the analysis to select the appropriate value. In order to remove the mass flow rate as an input, a new input was required that could uniquely define the operating condition to be analyzed. After a review of several options, it was determined that the appropriate case input is static pressure at the exit of the turbine. This parameter was selected as the ratio of inlet total pressure to exit static pressure ultimately sets the flow rate through the turbine and provides a unique solution for each value analyzed. This input change is implemented through the top-level solver and is discussed in more detail in the following section.

\section{Changes to the Solver Setup}

The final changes made to the OTAC model to enable analysis of choked flow involve modifications to the solver setup. As shown in Fig. 2, the default setup includes a solver for each BladeRow object and a top-level solver. The top-level solver was typically empty but could be added to by the user.

The first modification to the solver setup involves adding the inlet mass flow rate as an independent and an exit static pressure dependent equation (described by Eq. 11) to the top-level solver.

$$
P_{s, \text { exit }}=P_{s, \text { input }}
$$

The second change to the solver setup involves adding the choked flow constraint to the model. The NPSS Newton-Raphson solver allows for constraints to be added by attaching them to an existing dependent equation. When the constraint limit is violated, the constraint equation becomes active and replaces the dependent it is attached to in the solver. Based on the review of the flow physics presented previously, the flow angle dependent equation becomes invalid when the flow chokes as the exit flow angle is no longer determined by the blade exit metal angle and deviation model but is instead set to match the blade row exit static pressure.

The final change to the solver setup requires moving several independents and dependents from the blade row solvers to the top-level solver. These changes are required in order to put all coupled independents and dependents in the same solver. The independents which must be moved to the top-level solver are the exit Mach number and exit flow angle while the dependents to be moved are the exit area and exit flow angle. The constraint attached to the exit flow angle dependent is also moved as part of this process. This revised solver setup is shown in Fig. 5.

The result of making these changes to the solver setup is an OTAC model which will converge across all flow regimes. For case inputs that result in subsonic flow, the mass flow rate will be varied to match the exit 
static pressure. The exit flow angle and exit Mach number from each blade row are also varied to satisfy the target flow angle and exit area dependents. For case inputs that produce supersonic flow, the flow rate constraint of the limiting blade row is activated and the mass flow rate is varied to satisfy this constraint. The exit flow angle of that blade row is then free to vary to satisfy the exit static pressure dependent. The remaining independents and dependents are solved as in the subsonic case.

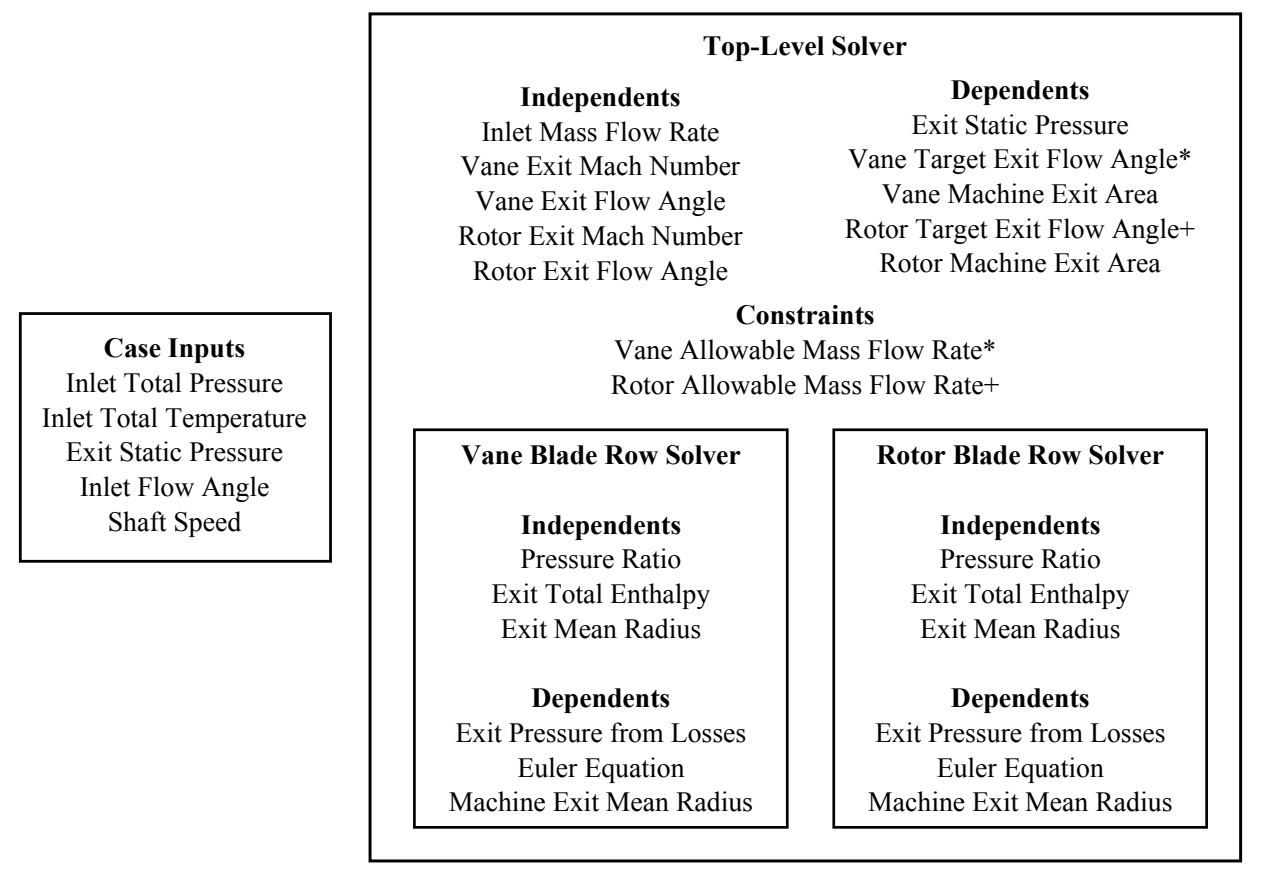

Figure 5. Case Inputs and Solver Setup for the Revised Single-Stage Turbine Model.

\section{Results}

The methodology described in the previous section has been successfully implemented in OTAC providing the ability to analyze turbine performance over a wide range of operating conditions. To demonstrate this improved capability, a number of single-stage and multi-stage turbines models were developed. These models were used to produce turbine performance maps which depict the flow and efficiency characteristics as a function of shaft speed and pressure ratio.

Before presenting the single-stage and multi-stage performance maps, a brief description of the modeling approach is required. For each of the turbine designs analyzed, the new capabilities described in Section IV were applied across all flow regimes (subsonic and supersonic). To create each corrected speed line on the map, the shaft speed and inlet total flow properties were held constant with changes to the exit static pressure used to move along the speed line. Finally, the turbine models presented in this section all use the Kacker-Okapuu loss model ${ }^{6}$ with corrections for incidence losses published by Moustapha, Kacker and Tremblay. ${ }^{7}$

\section{Single-Stage Results}

The first set of results provided in this paper focus on the performance characteristics of single-stage turbines. Three different single-stage turbine designs were modeled to demonstrate the new method and capture relevant turbine performance characteristics. The single-stage turbine performance maps also serve as a means of validating the revised method by comparing the flow characteristics of the generated maps to those found in published experimental maps.

The first single-stage turbine developed for this study was a low reaction design. The resulting design 
produced the performance map shown in Fig. 6. In this figure, the left plot shows the corrected flow as a function of the turbine pressure ratio and percent corrected speed, with speed lines ranging from $50 \%$ to $130 \%$ of the design speed. The corrected flow results show several interesting characteristics of the choked turbine flow. First, the pressure ratio required to choke the flow is different for each speed line, with higher pressure ratios required for higher speeds. Second, for this design all of the speed lines choke at the same corrected flow. This characteristic is the result of a design in which the choked passage is in the vane. ${ }^{8}$ Both of these characteristics match those found in experimental results (albeit for a different design) presented by Szanca and Schum ${ }^{9}$ in Fig. 7. The right plot of Fig. 6 depicts the turbine efficiency also as a function of pressure ratio and percent corrected speed. The figure shows that efficiency changes with speed and pressure ratio, with the lower speeds having a narrower range of pressure ratios that have high efficiency. These characteristics are generally similar to those found in empirical maps used in NPSS cycle models. However, the increases in efficiency observed at high pressure ratio values for the $90 \%$ to $130 \%$ speed lines do not match trends found in empirical NPSS maps and are the result of limitations of the selected loss model. At these points, the implemented model extrapolates outside the valid ranges in the calculations for shock and incidence losses. These results indicate that the selected loss model may be inadequate for modeling the performance at these extreme off-design conditions. Regardless of the validity of the loss models at these points, the results shown for this turbine indicate that the method developed for capturing choked flow in the turbine produce the correct trends.
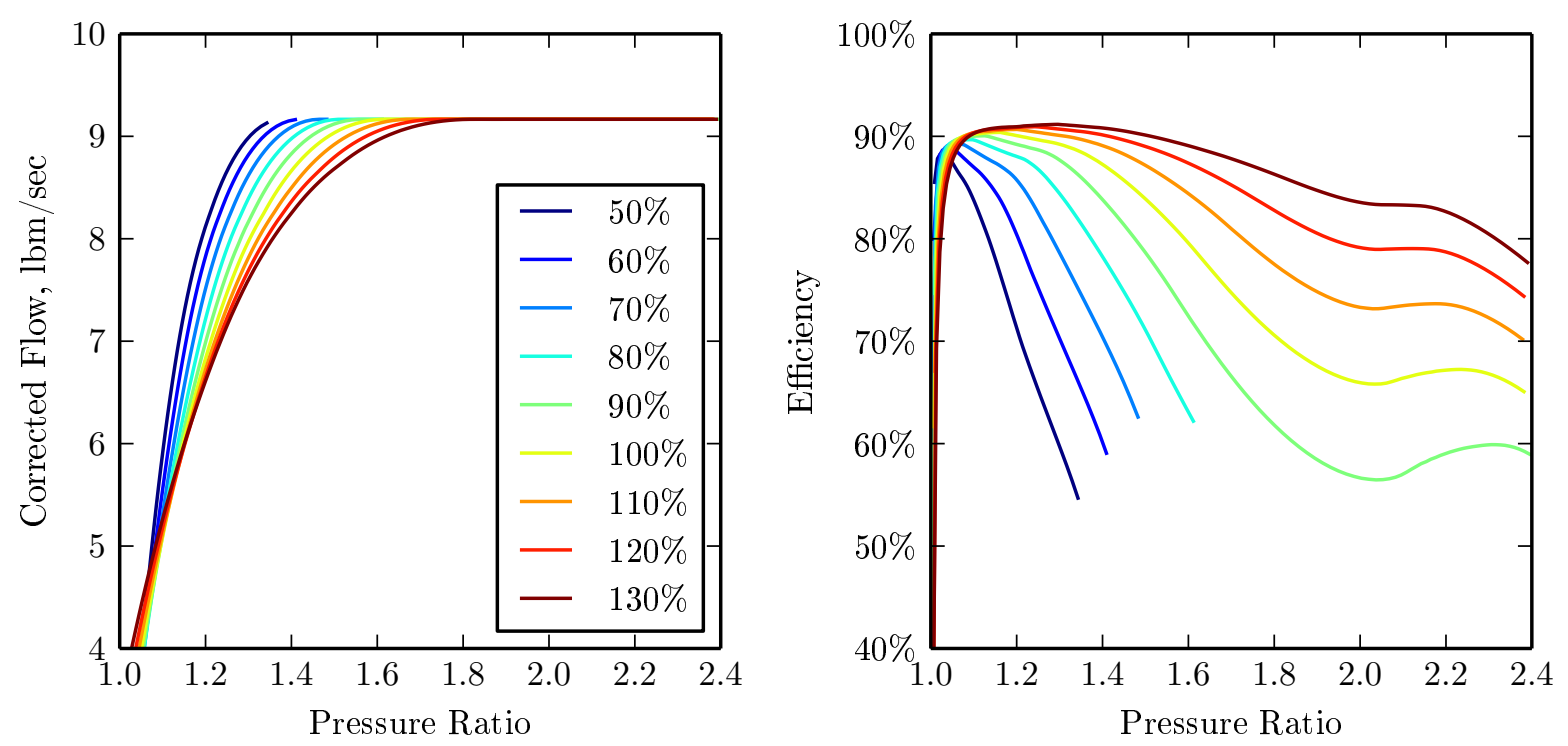

Figure 6. Single-Stage Turbine Map with Choked Vane Passages.

The second single-stage turbine design modeled had a higher degree of reaction and produced the performance map shown in Fig. 8. Similar to the first design, the performance map shows that the pressure ratio required to choke the flow varies with the corrected shaft speed. In addition, Fig. 8 shows that the maximum pressure ratio for each speed line varies which is not easily observed in performance map of the first design. The maximum pressure ratio point for each speed line corresponds to the limit load condition which is the maximum power that can be produced by the turbine at that speed. The key difference between this design and the first design is that the speed lines choke at different corrected flows. This characteristic indicates that the flow chokes in the rotor passage. ${ }^{8}$ The physical cause of this difference in the choked corrected flow for each speed line is the change in the rotor inlet relative pressure resulting from the change in the rotation speed. The characteristics of this turbine map again match those presented by Szanca and Schum $^{9}$ (again for a different design) as shown in Fig. 9 providing verification of the proposed method for capturing choked flow in the turbine. The efficiency characteristics shown in the right plot of Fig. 8 are similar to those identified for the first single-stage design.

Finally, the third single-stage turbine design developed for this study had an intermediate degree of 


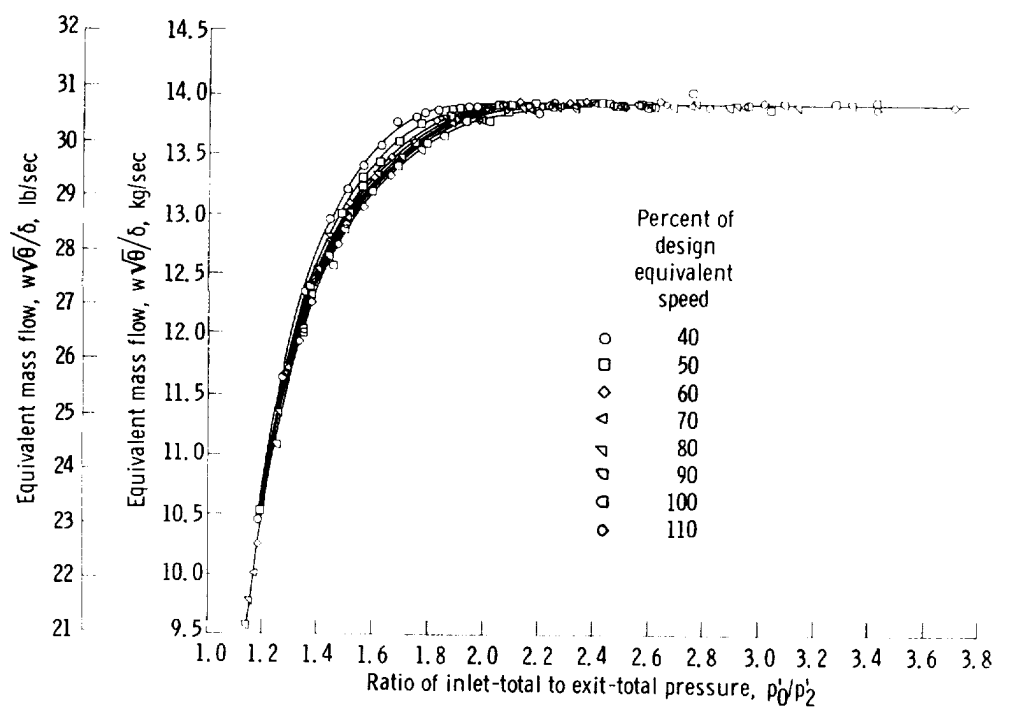

Figure 7. Experimental Results for a Single-Stage Turbine with a Choked Vane. ${ }^{9}$
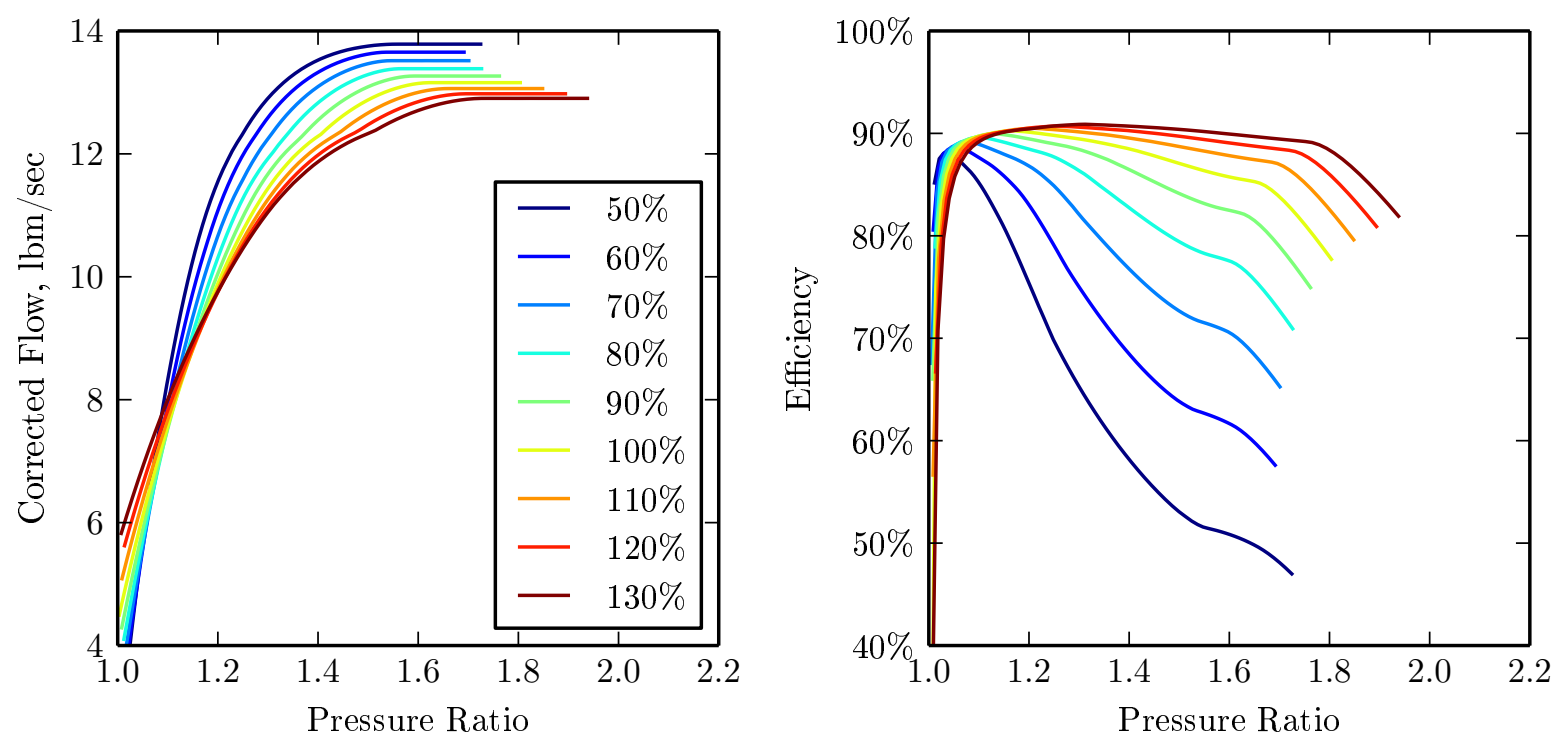

Figure 8. Single-Stage Turbine Map with Choked Rotor Passages. 


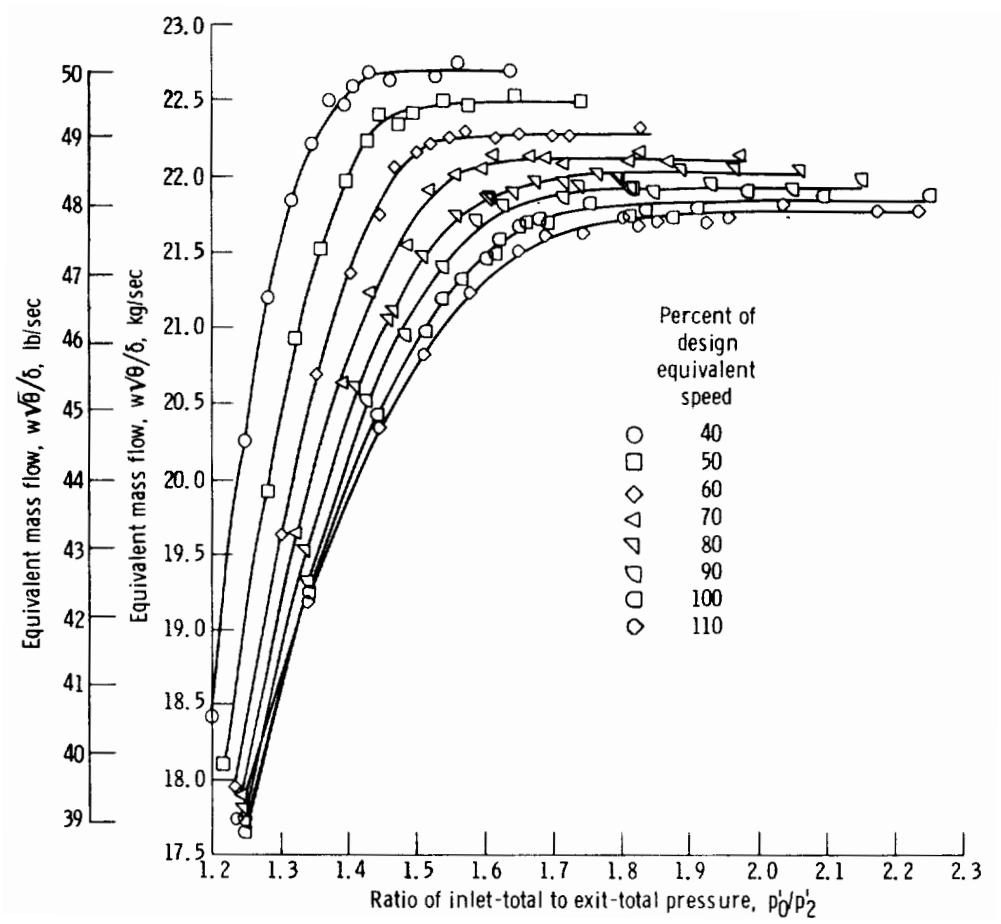

Figure 9. Experimental Results for a Single-Stage Turbine with a Choked Rotor. ${ }^{9}$

reaction. The resulting turbine performance map is shown in Fig. 10 and exhibits characteristics found in each of the first two designs. At low corrected speeds, the flow chokes in the vane resulting in the speed lines coalescing at the same corrected flow. However, at higher speeds the rotor passage chokes first resulting in a different choked flow for each speed line. This transition shows that the model is capable of automatically identifying the limiting flow passage and using that throat to set the allowable mass flow rate. Again, the efficiency characteristics shown this design are similar to those identified for the other two single-stage design reviewed in this section.

\section{Multi-Stage Results}

In addition to the single-stage turbine models examined in the previous section, several multi-stage models were created to evaluate the new method on more complex designs. The first multi-stage model considered consisted of two stages and produced the performance map shown in Fig. 11. The speed line trends exhibited in this map are similar to those of the second single-stage design previously presented. For this design, the speed lines each choke at a different corrected flow indicating the limiting passages is not the first vane. Further examination of the results show that the turbine chokes in the second rotor for all speeds except for $50 \%$. For operation at $50 \%$ speed, the throat in the second vane chokes first. This characteristic matches the results shown for the third single-stage model where the choked passage transitioned from the rotor to the vane at lower shaft speeds.

Lastly, a four-stage turbine model was created producing the performance map shown in Fig. 12. Again, the trends found in this map are indicative of a design where the flow chokes in a blade row other than the first vane as the speed lines choke at different corrected flow values. For this design, the limiting throat occurs in the last rotor for all speed lines. While these trend are similar to the single-stage and two-stage results shown previously, there is an important difference to note. For the four-stage design, the speed lines asymptote to the choked limit slowly compared to the single-stage design. A closer examination of the results shows that only a small segment of the speed line is choked before limit load is reached. This differs from the single-stage performance characteristics where a larger portion of the speed line is choked. 

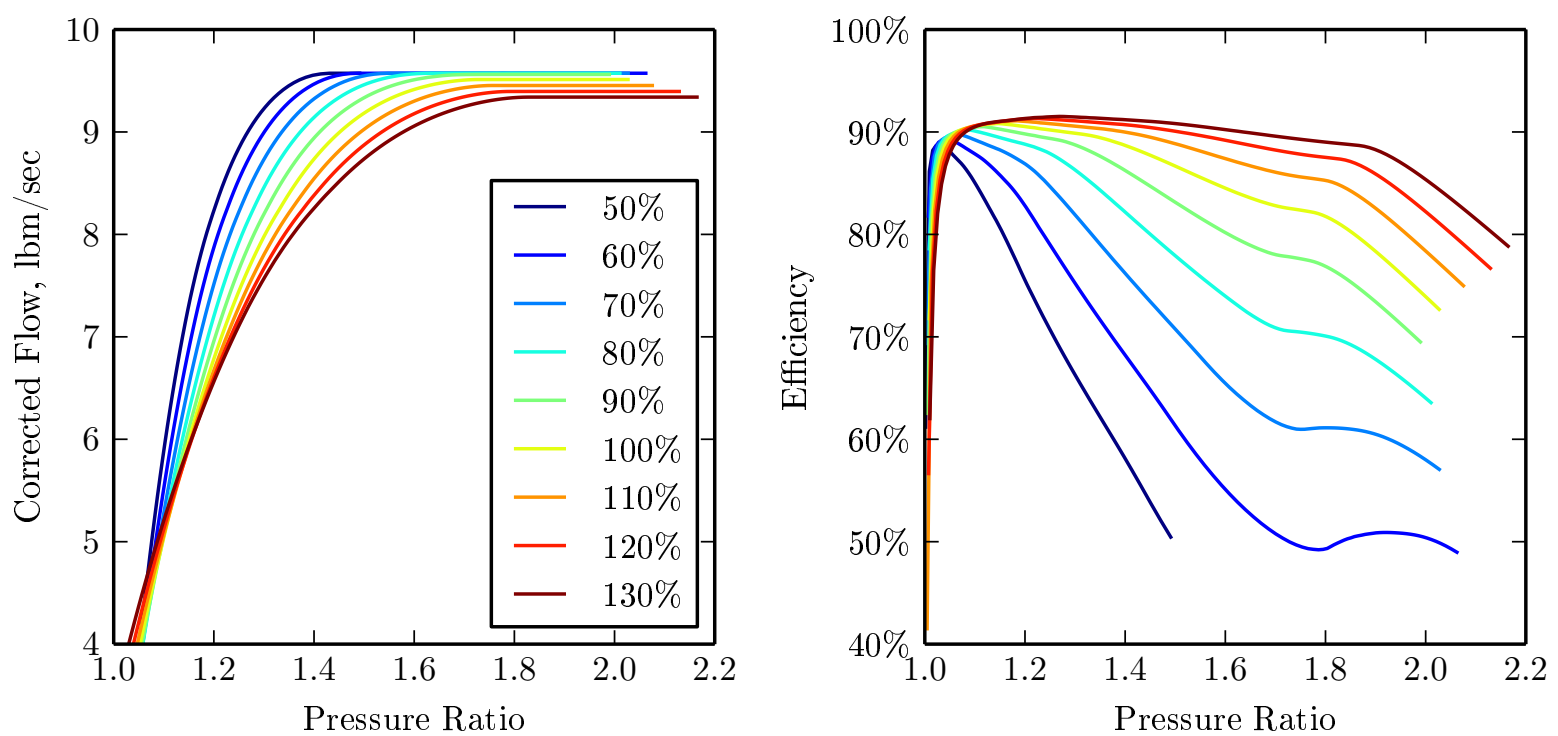

Figure 10. Single-Stage Turbine Map with Choked Passage Transitioning Between the Vane and Rotor.
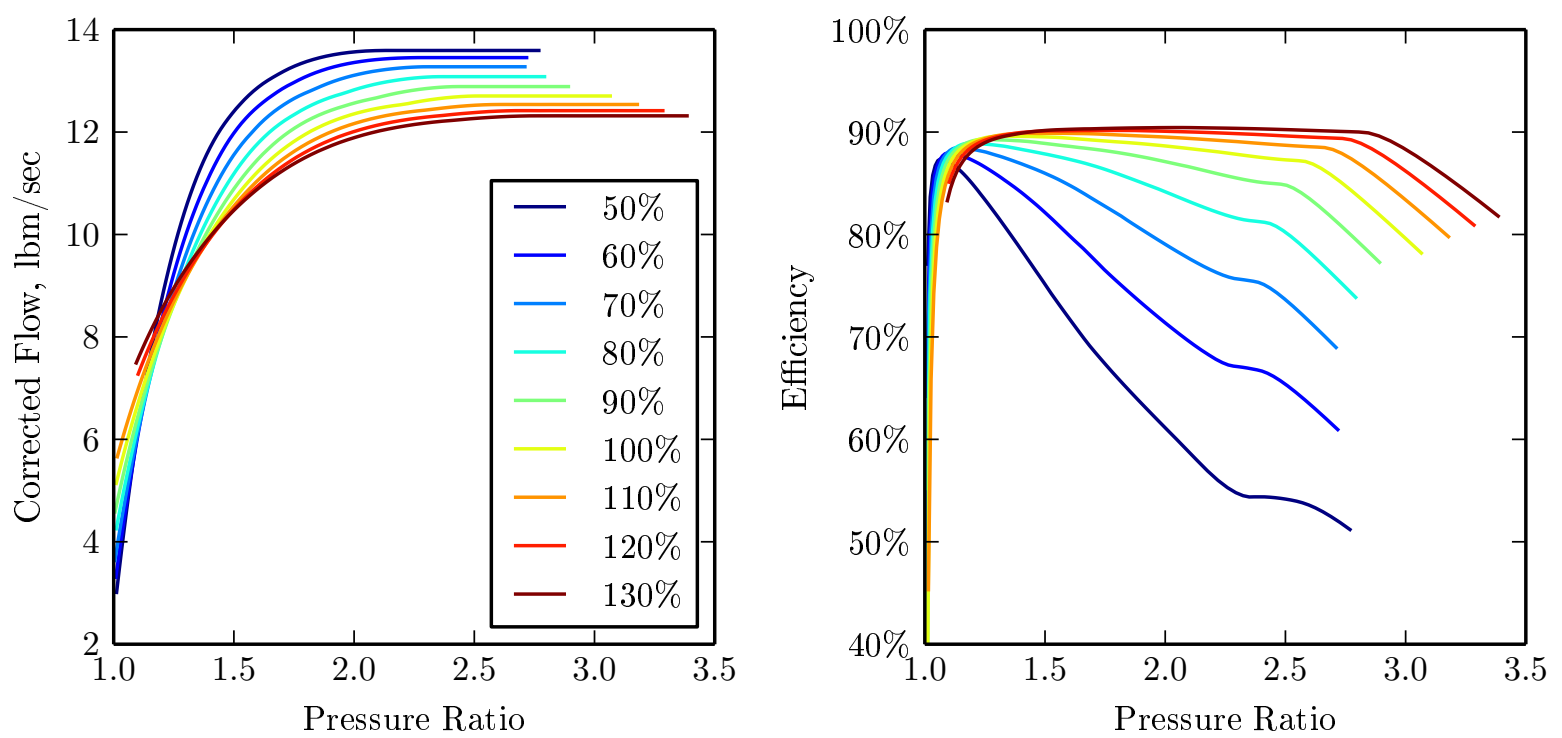

Figure 11. Two-Stage Turbine Performance Map. 

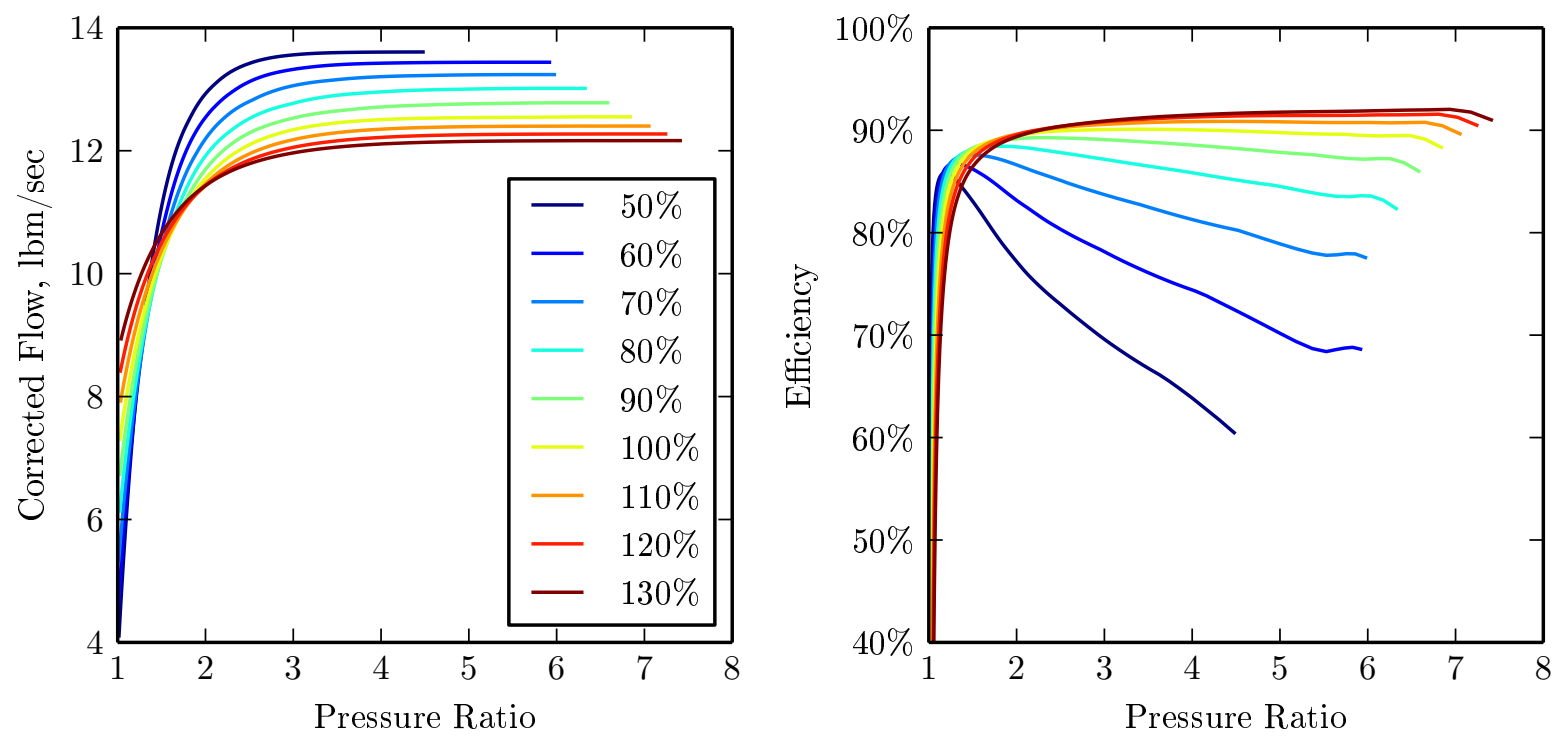

Figure 12. Four-Stage Turbine Performance Map.

\section{Conclusion and Future Work}

Prediction of turbomachinery performance is an important part of the engine conceptual design process. For many conceptual engine design studies, the prediction of turbomachinery performance is limited to scaling existing maps produced from experimental or simulation data. However, these existing maps may not be representative of new or unconventional turbomachinery designs and may undergo undesirable levels of scaling. Therefore, a new Object-Oriented Turbomachinery Analysis Code (OTAC) has been developed by NASA to enable the meanline and streamline analysis of compressor and turbine designs for conceptual design. While initial results validated OTAC for both compressors and turbines with subsonic flow, a limitation of the code was identified when relative Mach numbers became supersonic, which is of particular importance for turbine performance characterization.

In this paper, several modifications to OTAC were described which enable the analysis of turbines under supersonic relative flow conditions. Developing these improvements first required building an understanding of the relevant flow characteristics present in the choked turbine. After examining these characteristics, three specific improvements were made to the OTAC objects and baseline model setup to enable the analysis of these operating conditions. These changes included adding calculations to determine throat properties in each blade row, revising the model inputs, and modifying the OTAC solver setup.

With these improvements implemented in OTAC, several single-stage and multi-stage turbines were modeled to evaluate the modifications to the code. Results from these analyses were presented in the form of turbine maps. These maps demonstrated that the new modeling approach could be used to generate results across all flow regimes. Additionally, the maps depicted important performance characteristics associated with choked turbine flow such as which blade row served as the limiting passage. These characteristics were compared to those observed in maps found in literature showing that the method properly models turbine performance across all flow regimes.

The method presented in this paper provides an improved capability for OTAC. However, the method is currently limited to meanline analysis and requires extension to be applied to turbine streamline models. Additionally, a similar approach may need to be developed for OTAC to capture the choked flow region on compressor performance maps. The modeling approach for compressors will likely require similar changes to the OTAC model as those described in this paper. The primary challenge appears to be understanding the physical mechanisms which cause the flow to choke in the compressor. Once the flow characteristics are identified, the required changes to the OTAC objects, model inputs and solver setup can be determined and implemented. 


\section{Acknowledgments}

The work presented in this paper is supported by NASA's Revolutionary Vertical Lift Technology Project. The author would also like to thank Scott Jones of NASA Glenn Research Center as well as Dr. Jimmy Tai and Russell Denney of the Georgia Institute of Technology for their guidance of this work.

\section{References}

${ }^{1}$ Jones, S. M., "Development of an Object-Oriented Turbomachinery Analysis Code within the NPSS Framework," NASA TM-2014-216621, 2014.

${ }^{2}$ Claus, R. W., Evans, A. L., Lytle, J. K., and Nichols, L. D., "Numerical Propulsion System Simulation," Computing Sytems in Engineering, Vol. 2, No. 4, 1991, pp. 357-364.

${ }^{3}$ Jones, S. M., "Design of an Object-Oriented Turbomachinery Analysis Code: Initial Results," 22nd International Symposium on Air Breathing Engines, Phoenix, AZ, October 2015, ISABE2015-20015.

${ }^{4}$ Stratford, B. and Sansome, G., "The Performance of Supersonic Turbine Nozzles," Tech. Rep. Reports \& Memoranda No. 3273, Ministry of Aviation, June 1959.

${ }^{5}$ Glassman, A. J., "Estimating Turbine Limit Load," Contractor Report NASA/CR-191105, NASA, Lewis Research Center, Cleveland OH 44135, USA, April 1993.

${ }^{6}$ Kacker, S. C. and Okapuu, U., "A Mean Line Prediction Method for Axial Flow Turbine Efficiency," Journal of Engineering for Power, Vol. 104, 1982, pp. 111-119.

${ }^{7}$ Moustapha, S. H., Kacker, S. C., and Tremblay, B., "An Improved Incidence Losses Prediction Method for Turbine Airfoils," Journal of Engineering for Power, Vol. 112, 1990, pp. 267-276.

${ }^{8}$ Wilson, D. G. and Korakianitis, T., The Design of High-Efficiency Turbomachinery and Gas Turbines, Prentice-Hall, Upper Saddle River, New Jersey, 2nd ed., 1998.

${ }^{9}$ Szanca, E. M. and Schum, H. J., "Experimental Determination of Aerodynamic Performance," NASA/SP-290, Vol. 3, Lewis Research Center, Cleveland OH 44135, USA, 1975. 